\title{
Short-term Intraocular Pressure Spikes Induced by Pneumoperitoneum are Safe for the Optic Nerve in a Healthy Eye
}

Andrea Grosso, ${ }^{1,2}$ Piero Ceruti, ${ }^{3}$ Jonathan Crowston, ${ }^{4}$ Fabrizio Bert, ${ }^{5}$ Marco E Allaix, ${ }^{6}$ Alberto Bullano, ${ }^{6}$ Luca Grasso, ${ }^{6}$ Valentina Battaglino, ${ }^{7}$ Enrico Cavallo, ${ }^{6}$ Roberta Piccini Orthop, ${ }^{7}$ Giulio Piana Orthop, ${ }^{7}$ Beatrice Visentin Orthop, ${ }^{7}$ Roberta Siliquini ${ }^{5}$ and Mario Morino ${ }^{6}$

1. Centre for Macular Research and Allied Diseases, Torino, Italy; 2. Division of Ophthalmology, 'Santo Spirito' Hospital, Casale Monferrato, Italy; 3. Borgo Trento Hospital, University of Verona, Verona, Italy; 4. Ophthalmology and Department of Surgery, Centre for Eye Research Australia, University of Melbourne, Melbourne, Australia;

5. Department of Public Health Sciences and Pediatrics University of Torino, Torino, Italy; 6. Department of Surgical Sciences, University of Torino, Torino, Italy;

7. Department of Ophthalmology, Torino Eye Hospital, Torino, Italy

DOl: https://doi.org/10.17925/EOR.2018.12.1.61

$\mathrm{T}$ he aim of this study was to determine the effects of the pneumoperitoneum used in colorectal procedures on peripapillary retinal nerve fibre layer (RNFL) thickness. We also analysed the dynamics of ocular anterior segment structures. In this prospective clinical pilot study, 29 patients aged between 45 and 85 years old underwent laparoscopic surgery for selected colorectal diseases. The pneumoperitoneum induced a mean intraocular pressure (IOP) increase of $4 \mathrm{mmHg}$. Visual acuity, IOP, corneal thickness, angle opening distance (AOD) and the trabeculo-iris space area (TISA) at 500 microns and 750 microns anterior to the scleral spur, RNFL thickness and visual field were measured before and after surgery using Sloan charts, Visante ${ }^{\mathrm{TM}}$ optical coherence tomography (OCT) (Zeiss, Oberkochen, Germany), Spectralis ${ }^{\oplus}$ OCT (Heidelberg Engineering, Heidelberg, Germany) and Humphrey Visual Field System ${ }^{\oplus}$ (Zeiss, Oberkochen, Germany). Patients were categorised in two groups to understand the behaviour of IOP: in group one $(n=17)$, patients were in Trendelenburg position; in group two ( $n=12)$, patients were in reverse Trendelenburg position. The IOP was measured during surgery by the Icare ${ }^{\circledast}$ Tonometer (Icare, Vantaa, Finland) according to a standardised protocol; the IOP was measured before and after surgery by Goldmann applanation tonometer (Haag-Streit, Köniz, Switzerland). Patients with previous diagnosis of both open or angle-closure glaucoma or anterior segment anomalies were excluded. No statistically significant differences in increase of IOP were found between the two groups $(p>0.05)$; no statistically significant differences in RNFL thickness, visual field, AOD and TISA were found before and after surgery in all patients $(p>0.05)$. In our study we have demonstrated that a mean IOP increase of $4 \mathrm{mmHg}$ over a short period of time is safe for a healthy optic nerve.

\section{Keywords}

Intraocular pressure, laparoscopic surgery, peripapillary retinal nerve fibre layer, pneumoperitoneum, optical coherence tomography, visual loss

Disclosure: Andrea Grosso, Piero Ceruti, Jonathan Crowston, Fabrizio Bert, Marco E Allaix, Alberto Bullano, Luca Grasso, Valentina Battaglino, Enrico Cavallo, Roberta Piccini Orthop, Giulio Piana Orthop, Beatrice Visentin Orthop, Roberta Siliquini and Mario Morino have nothing to disclose in relation to this article.

Acknowledgements: Acknowledgements to the ICARE staff and in particular to Daniel Borrego; their cooperation was fundamental to make our project possible. Clinical advice from Professor Luciano Quaranta, Ophthalmologist in Chief, Spedali Civili di Brescia, was appreciated. We thank Dr Marco Amisano, Chief of General Surgery at Santo Spirito

Hospital in Casale Monferrato for sharing his experience in laparoscopic surgery with us, and for his encouragement.

Review Process: Double-blind peer review.

Compliance with Ethics: All procedures were followed in accordance with the responsible committee on human experimentation and with the Helsinki Declaration of 1975 and subsequent revisions. Informed consent was received from all patients involved in this study. All patients signed a study-specific informed consent form. The study protocol received the approval of the Hospital Ethical Committee (Protocol Number 0026385).

Authorship: All named authors meet the International Committee of Medical Journal Editors (ICMJE) criteria for authorship of this manuscript, take responsibility for the integrity of the work as a whole, and have given final approval to the version to be published.

open Access: This article is published under the Creative Commons

Attribution Noncommercial License, which permits any non-commercial use, distribution, adaptation and reproduction provided the original author(s) and source are given appropriate credit. (c) The Authors 2018.

Received: 6 February 2018

Accepted: 3 April 2018

Citation: European Ophthalmic Review, 2018;12(1):60-5

Corresponding Author: Andrea Grosso, Centre of Macular Research, Via Roma 73, San Mauro Torinese, Italy. E: 78andreagrosso@gmail.com

Support: No funding was received in the publication

of this article.
It is well known that blindness can occur after laparoscopic surgery as a result of severe head-down tilt for $>6-7$ hours. Among possible causes, a relationship between intraocular pressure (IOP) and optic nerve damage has been suggested. ${ }^{1}$ We previously described that IOP, detected by the Icare ${ }^{\circledast}$ Pro $^{\circledast}$ Tonometer (Icare, Vantaa, Finland), may rise after pneumoperitoneum during colorectal surgery. We have also demonstrated that patients in Trendelenburg positioning showed a greater increase in IOP after pneumoperitoneum induction when compared to patients without Trendelenburg positioning. ${ }^{2}$ However, little is known about the mechanisms underlying the IOP rise during pneumoperitoneum. Furthermore, it is not clear if IOP elevation may cause loss of optic nerve fibres.

The purpose of this prospective clinical study was to evaluate the changes in peripapillary retinal nerve fibre layer (RNFL) thickness before and after laparoscopic surgery. As an ancillary aim we have analysed the dynamics of anterior segment structures.

\section{Methods}

Study design and population

This prospective clinic-based study was conducted at the Digestive, Colorectal and Oncological Surgery Unit of the University of Torino, Italy. Inclusion criteria included: age between 45 and 85 years, body mass index (BMI) $\leq 35 \mathrm{~kg} / \mathrm{m}^{2}$, American Society of Anaesthesiologists (ASA) classification I to III and surgical indication of laparoscopic colorectal procedure. Exclusion criteria included: established diagnosis of glaucoma with topical therapy, documented increased IOP, recent ( $\leq 6$ months) eye infections and anterior segment abnormalities. 
All patients signed a study-specific informed consent form. The study protocol received the approval of the Hospital Ethical Committee (Protocol Number 0026385)

\section{Data collection}

For each patient enrolled in the study, the following demographic and clinical data were collected: gender, age, BMI and smoking habits. Several ophthalmological variables were registered. The peripapillary RNFL thickness was measured by Spectralis ${ }^{\circledR}$ SD-optical coherence tomography (OCT) (Heidelberg Engineering, Heidelberg, Germany), the iridocorneal angle measurement was performed by Visante ${ }^{\circledast}$ OCT (Anterior Segment Imaging System, Zeiss, Oberkochen, Germany) and the visual field was obtained by Humphrey Visual Field System ${ }^{\circledR}$ (Zeiss, Oberkochen, Germany). Visual acuity was assessed on Sloan charts. Best corrected visual acuity was registered.

Patients were categorised into two groups to understand the behaviour of IOP: in group one $(n=17)$ patients were in Trendelenburg position, in group two $(n=12)$ patients were in reverse Trendelenburg position. All laparoscopic procedures were performed by a single experienced surgeon (MM). Our surgical techniques have been described elsewhere. ${ }^{2,3}$ The operative time was also registered. The tonometer used to measure IOP during surgery was the Icare Pro Tonometer. 4,5 IOP was measured in both eyes at eight time points by a specifically trained medical student $(E C)$ and trained surgeons (MA, LG, AB) according to a standardised protocol described in our previous report. ${ }^{2}$ To define increased IOP, we chose a predetermined arbitrary increase in pressure of $\geq 5 \mathrm{mmHg}$ compared with the preoperative value.

The baseline preoperative IOP value and the IOP 1 month after surgery was measured by an ophthalmologist (VB) using Goldmann applanation tonometry (Haag-Streit, Köniz, Switzerland), with patients in a horizontal, lying position. Two measures were performed in all patients and IOP was defined as the mean value of these.

\section{Ophthalmological examinations}

All patients underwent visante OCT before and 1 month after surgery. OCT allows imaging of the anterior segment, specifically the angle structures, the anterior chamber and cornea. The reliability of measurements with Visante OCT is operator-dependent; therefore all measurements were recorded by trained orthoptists (BV, GP, RP) between 08:00 and 12:00 in ambient room light conditions. ${ }^{6,7}$ The angle opening distance (AOD) and the trabeculo-iris space area (TISA) at 500 microns and 750 microns anterior to the scleral spur were measured both pre- and post-surgery. Central corneal thickness (CCT) was also obtained by Visante OCT.

The measurement of peripapillary RNFL was performed by the Spectralis OCT; the RNFL thickness values obtained were compared with those included in the spectralis OCT database (qualitative analysis) and classified as normal versus abnormal. Differences in thickness of RNFL before and 15 days to 1 month after surgery were also analysed (quantitative analysis) in all patients. All patients underwent Humphrey Visual Field (24-2 Swedish Interactive Threshold Algorithm standard) before and after surgery.

\section{Anaesthesia protocol}

The anaesthesia protocol was standardised for the drugs used during the procedure and described in our previous publication. ${ }^{2}$
Table 1: Patients characteristics classified according to the use or not of the Trendelenburg position during surgery $(n=29)$

\begin{tabular}{|l|l|l|l|}
\hline & $\begin{array}{l}\text { Trendelenburg } \\
\% \mathrm{n}\end{array}$ & $\begin{array}{l}\text { Reverse Trendelenburg } \\
\% \mathrm{n}\end{array}$ & p-value \\
\hline Gender & & & 0.132 \\
\hline Male & $47.1(8)$ & $52.9(9)$ & \\
\hline Female & $75.0(9)$ & $25.0(3)$ & 0.795 \\
\hline Mean age & 54.1 & 55.4 & 0.248 \\
\hline BMI, Kg/m² & & & \\
\hline $18.5-24.9$ & $50.0(7)$ & $50.0(7)$ & \\
\hline $25.0-29.9$ & $50.0(4)$ & $50.0(4)$ & 0.09 \\
\hline$\geq 30.0$ & $85.7(6)$ & $14.3(1)$ & \\
\hline Tobacco smoke & & & \\
\hline Yes & $80.0(8)$ & $20.0(2)$ & \\
\hline No & $47.4(9)$ & $52.6(10)$ & \\
\hline
\end{tabular}

Operative time: minutes. $B M I=$ body mass index.

\section{Statistical analysis}

The present study was considered a 'pilot study', thus no a priori sample size calculation was performed. Descriptive analyses were performed using frequencies, percentages and frequency tables for categorical variables. For the bivariate analysis, chi-square tests were performed to evaluate differences for categorical variables. Results are expressed as adjusted odds ratio (OR) with 95\% confidence interval (Cl) and the goodness of fit of the model was assessed by the Hosmer-Lemeshow test. A two-tailed $p$-value of 0.05 was considered significant for all analyses, which were carried out using Stata, version 10.1 (Stata Corp., College Station, TX, USA, 2007).

The normative values of RNFL thickness may be different according to the type of instrument. In our study we have used the database provided by the Spectralis OCT. The increase of IOP was analysed separately for group one and group two.

\section{Results}

This prospective clinical study enrolled 29 patients, including 17 men and 12 women. Patients were categorised into two groups to understand the behaviour of IOP: in group one $(n=17)$ patients were in Trendelenburg position, in group two $(n=12)$ patients were in reverse Trendelenburg position (Table 1).

In group one the operative time was $88.29 \pm 39.98$ minutes and in group two the operative time was $89.28 \pm 35.45$ minutes $(p=0.862)$.

The operations performed by laparoscopy were: five anti-reflux plastic (17.2\%), five sleeve gastrectomy (17.2\%), five left hemicolectomy (17.2\%), four cholecystectomies (13.8\%), four total mesorectal excision (13.8\%), three oesophageal myotomies (10.3\%), two right hemicolectomy (6.9\%) and one left surrenectomy (3.4\%). Ten of the 29 procedures performed required lateral inclination to the right side (34.5\%), 13 induced significant lateral inclinations (44.8\%) and only six required a lateral head inclination on the left side (20.7\%).

No statistically significant differences in increase of IOP during surgery were found between the two groups ( $p>0.05$ ) as shown by Figure 1 and Table 2. In addition, no statistically significant differences in increase of 
Figure 1: Mean intraocular pressure trend according to the use of Trendelenburg or reverse Trendelenburg position during surgery

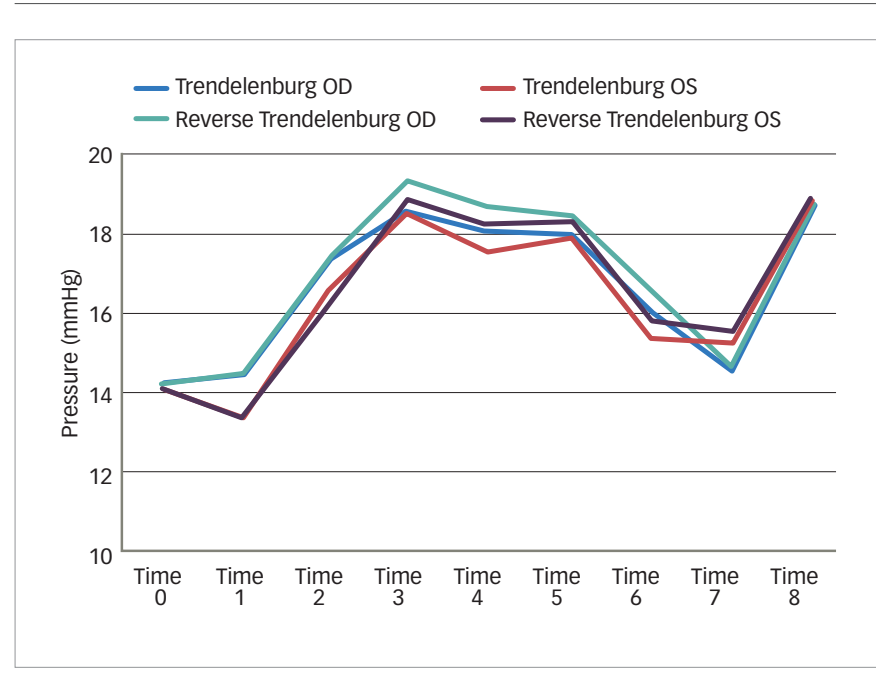

Time 0: at patient awakening 6-1 h before surgery; Time 1: 5 minutes after induction of anaesthesia; Time 2: 5 minutes after induction of pneumoperitoneum; Time 3:

5 minutes after placement of the patient in Trendelenburg position; Time 4: 20 minutes after placement of the patient in Trendelenburg position; Time 5: 45 minutes after placement of the patient in Trendelenburg position; Time 6:5 minutes after abdominal desufflation; Time 7: 5 minutes before patient extubation; Time 8: 48 hours after surgery. $O D=$ right eye; $O S$ = left eye.

Table 2: Comparison between Trendelenburg and reverse Trendelenburg position during surgery in patients with increased intraocular pressure

\begin{tabular}{|l|l|l|l|}
\hline \multicolumn{1}{|l|}{$\begin{array}{l}\text { Trendelenburg } \\
\%(n=17)\end{array}$} & $\begin{array}{l}\text { Reverse Trendelenburg } \\
\%(n=12)\end{array}$ & $p$-value \\
\hline IOP increase after the induction of anaesthesia & 0.429 \\
\hline Right eye & $0.0(0)$ & $8.3(1)$ & \\
\hline Left eye & - & - & \\
\hline IOP increase after the induction of pneumoperitoneum & \\
\hline Right eye & $31.3(5)$ & $41.6(5)$ & 0.698 \\
\hline Left eye & $37.5(6)$ & $33.3(4)$ & 0.864 \\
\hline
\end{tabular}

IOP increase 5 minutes after the induction of pneumoperitoneum

\begin{tabular}{|l|l|l|l|}
\hline Right eye & $62.5(10)$ & $58.3(7)$ & 0.867 \\
\hline Left eye & $25.0(4)$ & $33.3(4)$ & 0.691 \\
\hline
\end{tabular}

IOP increase $\mathbf{2 0}$ minutes after the induction of pneumoperitoneum

\begin{tabular}{|l|l|l|l|}
\hline Right eye & $50.0(8)$ & $19.0(4)$ & 0.620 \\
\hline Left eye & $37.5(6)$ & $66.6(8)$ & 0.252 \\
\hline
\end{tabular}

IOP increase $\mathbf{4 5}$ minutes after the induction of pneumoperitoneum

\begin{tabular}{|l|l|l|l|}
\hline Right eye & $37.5(6)$ & $41.6(5)$ & 0.867 \\
\hline Left eye & $31.3(5)$ & $33.3(4)$ & 0.770 \\
\hline
\end{tabular}

IOP increase after abdominal desufflation

\begin{tabular}{|l|l|l|l|}
\hline Right eye & $18.7(3)$ & $50.0(6)$ & 0.114 \\
\hline Left eye & $18.7(3)$ & $16.6(2)$ & 0.722 \\
\hline
\end{tabular}

IOP increase before patient extubation

\begin{tabular}{|l|l|l|l|}
\hline Right eye & $0.0(0)$ & $41.6(5)$ & 0.008 \\
\hline Left eye & $0.0(0)$ & $16.6(2)$ & 0.175 \\
\hline IOP increase 48 hours after surgery & \\
\hline Right eye & $25.0(4)$ & $19.0(4)$ & 0.691 \\
\hline Left eye & $43.7(7)$ & $25.0(3)$ & 0.434 \\
\hline
\end{tabular}

Increased intraocular pressure was defined as a rise of pressure of $\geq 5 \mathrm{mmHg}$ compared to the pre-operative value. Data are expressed as percentages and number. $I O P=$ intraocular pressure.
IOP were found before and after surgery in all patients: these results are of pivotal importance because it means that increase of IOP is a transient, reversible phenomenon. We did not find any statistically significant associations between the time in Trendelenburg position and the increase in IOP (right eye: OR 0.98; 95\% Cl 0.97-1.02; $p=0.786$, left eye: OR 1.00; 95\% $\mathrm{Cl} 0.98-1.03 ; p=0.668$ ).

Table 3 summarises the clinical findings in the pre- and post-operative ophthalmological examination in all patients. Twenty-six patients underwent pre-operative RNFL thickness measurement; 29 patients were screened at the end of surgery.

No statistically significant differences in RNFL thickness (both for qualitative as well as quantitative analysis), visual field, AOD and TISA were found before and after surgery in all patients ( $p>0.05)$. In Table 3, we have only provided data from the qualitative analysis to make this more readable.

No post-operative complications of respiratory nature or abnormalities in ocular hemodynamics were reported.

\section{Discussion}

Our study analysed the effects of the $12-14 \quad \mathrm{mmHg}^{\mathrm{CO}_{2}}$ pneumoperitoneum routinely used in colorectal procedures on peripapillary RNFL, which leads to a mean IOP increase of $4 \mathrm{mmHg}$.

To our knowledge this study is the first to show RNFL thickness changes following laparoscopic surgery for selected colorectal diseases. We have compared the RNFL changes in a series of consecutive patients before and after surgery: the differences were not statistically significant.

The choice of a cut-off in RNFL thickness measure was controversial. Miki et al. have proposed a categorisation in a selected group composed by glaucoma suspected eyes but these values cannot be generalised. ${ }^{8}$ Recently, Govetto et al. published a paper exploring the association between vitrectomy and glaucoma. ${ }^{\text {They }}$ compared RNFL values of vitrectomised versus non-vitrectomised eyes without establish a threshold value. 9,10

In this study we have made two different analyses: 1) a qualitative analysis, by comparing the values obtained in the OCT report with the normative data in Spectralis database (Figure 2 and Table 3); and 2) a quantitative analysis by providing a comparison between the measures of RNFL thickness before and after surgery. Many patients may have normal RNFL thickness profiles that, although not pathologic, deviate from normative RNFL thickness values. It was suggested that interocular difference in average RNFL thickness, measured with optical coherence tomography, should not differ by more than 9-12 mm. A higher difference may suggest early glaucomatous damage. 11-14 Quigley and Sommer reported that 12-63\% of optic nerve fibres can be lost before glaucomatous visual field defects are detected by routine kinetic perimetry. ${ }^{14}$ Several recent studies have confirmed that serial OCT RNFL measurements can reliably determine changes of $10 \%$ or greater and that relatively small percent changes in peripapillary RNFL thickness may be a reliable indicator of glaucoma. ${ }^{15}$

In our study we did not find changes in linear measures of anterior chamber (TISA and AOD) before and after surgery (Figure 3). The inter-observer variability on images taken throughout different times is unacceptable, but it gradually improves if we consider the repeated 
Table 3: Main ophthalmological data

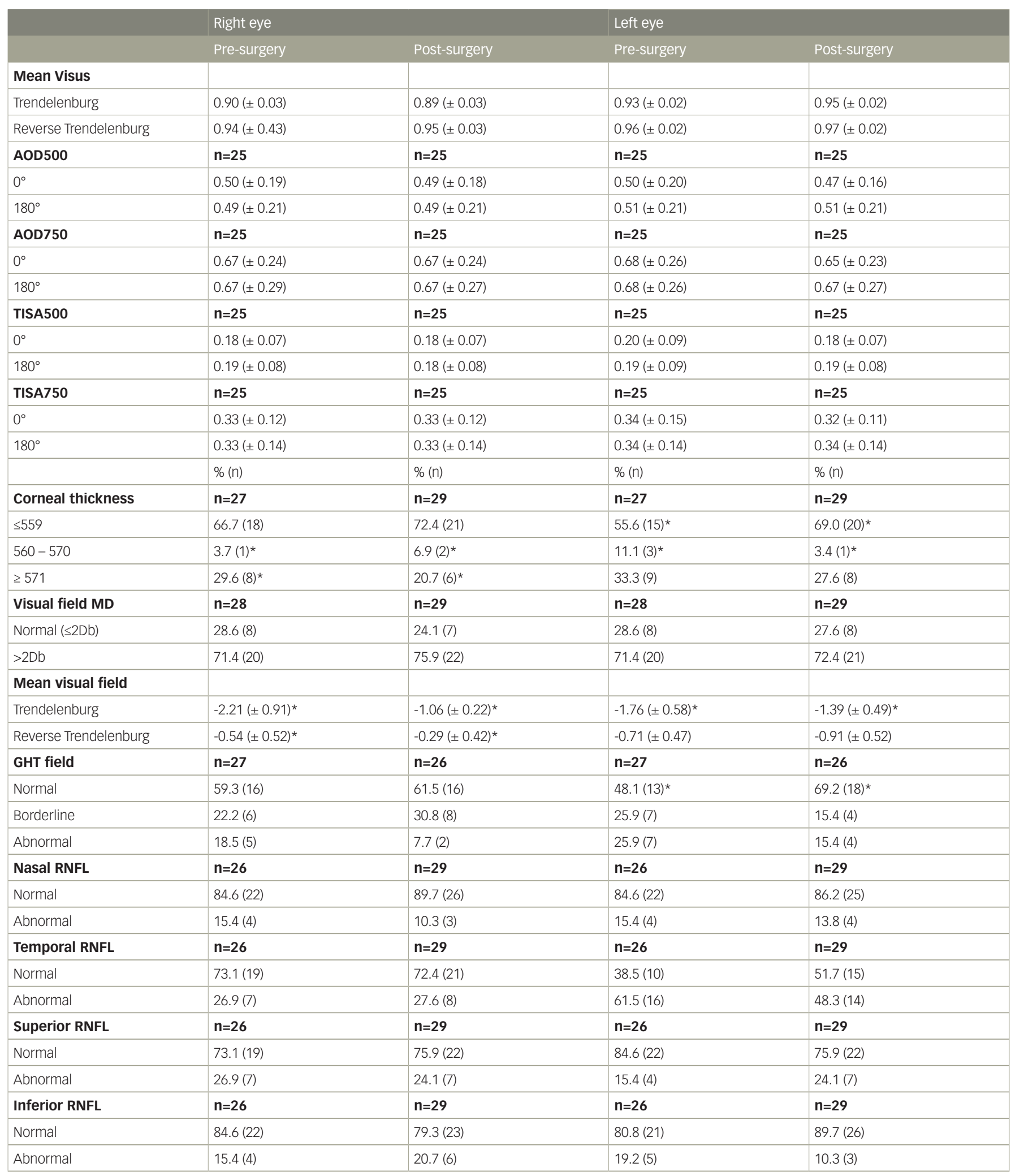

${ }^{*} p>0.05$. Only data from qualitative analysis is provided for readability.

$A O D=$ angle opening distance; $G H T=$ glaucoma hemifield test; $M D=$ mean deviation; $R N F L=$ retinal nerve fibre layer; $T I S A=$ trabeculo-iris space area.

inter-observer evaluation of the same image and even more if we consider the intra-observer variability on images taken in different times or better on the same image. Therefore, the presence of a trained operator is very important for consistent results. In this study the same operators (RP, VB, BV, GP) performed the Visante OCT measurements during the morning.
The behaviour of IOP in our study, in contrast with our previous report $^{2}$ and literature, ${ }^{16-19}$ remains unanswered. We could expect that patients in the Trendelenburg position would show a greater increase in IOP after pneumoperitoneum induction than patients in the reverse Trendelenburg position. ${ }^{2}$ The small sample size in our study weakens the conclusions of our analysis. It is also not clear whether 
Figure 2: OCT scans show qualitative analysis of RNFL thickness in the same patient before and following general surgery
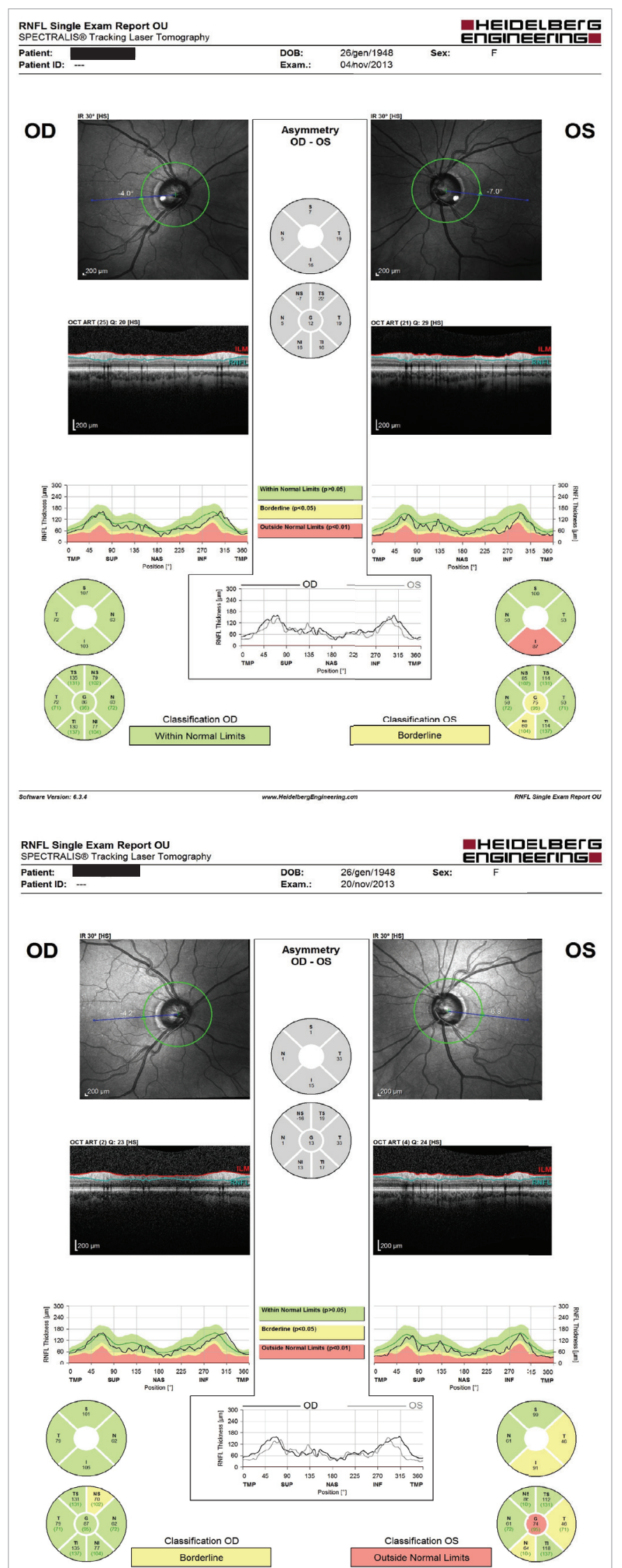
Borderline

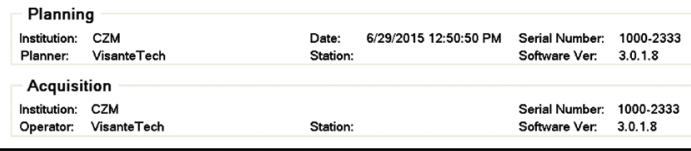

Report Date: 6/29/2015 12:51:01 PM Page: 1
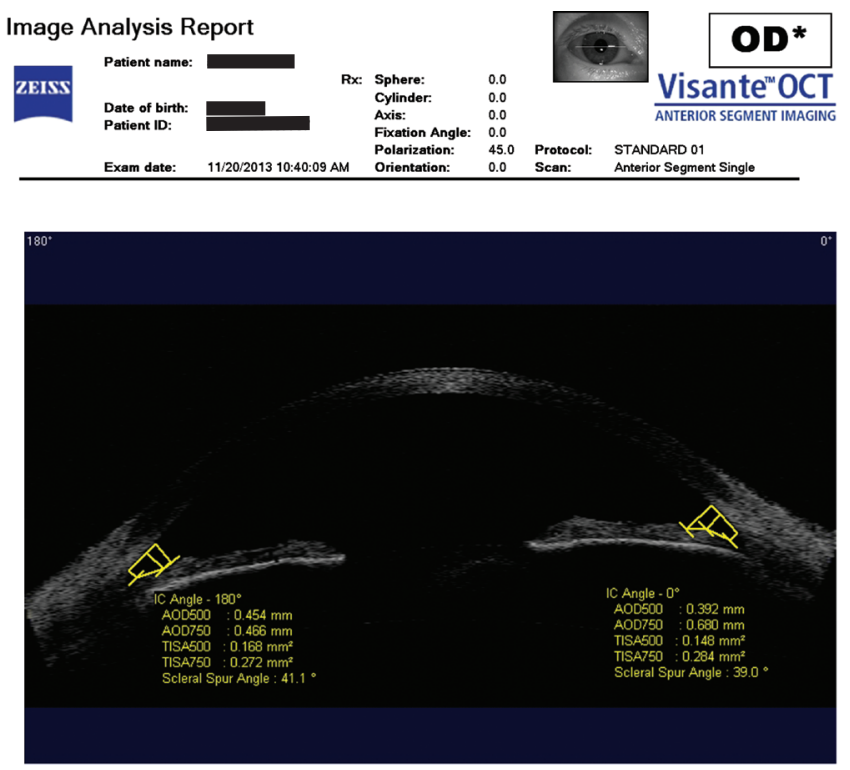
stress-signalling pathways induced by an increase of IOP may lead to long-term damage. Patients need to be informed about the opportunity of an ophthalmological examination after surgery and on a regular basis every year to be reassured that they do not carry the risk of glaucoma. Furthermore, as shown in our protocol, a single IOP measurement might not adequately approximate the IOP fluctuations; efforts are being made by the ophthalmological community towards continuous 24-hour monitoring of IOP.20

Strengths of our study included exclusion of patients with glaucoma and ocular pathology, and only patients with healthy eyes were considered. The ophthalmological follow up was extended to 1 month after surgery.

A limit of the study was the sample size, thus reducing the power of the study and the consistency of results. We were also concerned about the heterogeneous group of patients studied. We feel it would have been better if only those patients who had prolonged laparoscopic colorectal surgery were included, rather than adding in many short duration operations that have little head-down tilt or even have head-up tilt. Further studies are required, involving larger samples and different abdominal surgical procedures with both low and high insufflation pressures. We did assess RNFL thickness at one-month post-operation as an arbitrary end point as no patient had symptoms or very high pressure in this series. The prevalence of perioperative visual loss (POVL) after colorectal surgery was shown to be low (1.24 incidence per 10,000) in a large analysis. ${ }^{21}$ Unfortunately, the clinical impression among surgeons is that the development of POVL remains unpredictable and is not related to any particular hospital characteristic or degree of surgical experience.
It is known that pneumoperitoneum may cause an increase of IOP in all patients who undergo laparoscopic surgery. The IOP increase is reversible and does not determine optic nerve damage for short duration operations that have little head-down tilt or even have head-up tilt in healthy eyes. However, it is a different story for patients who had prolonged laparoscopic colorectal surgery. According to literature, cases of blindness after laparoscopic surgery have occurred in those operations that have had severe head-down tilt for at least 6 hours. ${ }^{1}$ We recommend an ophthalmic consultation as a part of routine follow up in these patients. Although the IOP increase of $4 \mathrm{mmHg}$ found in our study is unlikely to be dangerous for a healthy optic nerve, it might be significantly higher when high pneumoperitoneum insufflation pressures are used, such as in gynaecologic and urologic procedures, ${ }^{18,19}$ with potential damage not only in patients with advanced glaucoma but also in normal eyes.

Our data may legitimate the need for a risk assessment model to help identify patients - such as glaucoma patients - at risk of worsening following laparoscopic colorectal surgery, particularly in surgeries where patients need to be placed in the Trendelenburg position for a long time. There will be little harm from a fluctuation of $4 \mathrm{mmHg}$ during the course of surgery unless the blood pressure falls substantially at the same time. Fluctuations may be much larger if outflow facility is impaired as is often the case in glaucoma. Patients with a history of glaucoma need to be screened in every case of laparoscopic surgery in liaison with ophthalmologists. Patients with angle closure glaucoma need a specific preparation with eye drops and, depending on the case, systemic acetazolamide before the surgical procedure and careful analysis of optic nerve clinically and by imaging. ${ }^{22}$
1. Pinkney TD, King AJ, Walter $C$, et al. Raised intraocular pressure (IOP) and perioperative visual loss in laparoscopic colorectal surgery: a catastrophe waiting to happen? A systematic review of evidence from other surgical specialities. Tech Coloproctol. 2012;16:331-5.

2. Grosso A, Scozzari G, Bert F, et al. Intraocular pressure variation during colorectal laparoscopic surgery: standard pneumoperitoneum leads to reversible elevation in intraocular pressure. Surg Endosc. 2013;27:3370-6.

3. Morino M, Parini U, Girando G, et al. Laparoscopic total mesorecterision: Ann surg. 2003;237:335-42.

4. Scuderi GL, Cascone NC, Regine F, et al. Validity and limits of the rebound tonometer (ICarem): clinical study. Eur J Ophthalmol. 2011;21:251-7.

5. Brusini P, Salvetat ML, Zeppieri M, et al. Comparison of ICare tonometer with Goldmann applanation in glaucoma patients. J Glaucoma. 2006; 15:213-7.

6. Radhakrishnan S, See J, Smith SD, et al. Reproducibility of anterior chamber angle measurements obtained with anterior segment optical coherence tomography. Invest Ophthalmol Vis Sci. 2007;48:3683-88.

7. Tello C, Liebmann J, Potash SD, et al. Measurement of ultrasound biomicroscopy images: intraobserver and interobserver reliability. Invest Ophthalmol Vis Sci. 1994:35:3549-52.
8. Miki A, Medeiros FA, Weinreb RN, et al. Rates of retinal nerve fibre layer thinning in glaucoma suspect eyes. Ophthalmology. 2014;121:1350-8

9. Govetto A, Domíniguez R, Landaluce ML, et al. Prevalence of open angle glaucoma in vitrectomized eyes: a cross-sectional study. Retina. 2014;34:1623-9.

10. Grosso A. Re: Prevalence of open angle glaucoma in vitrectomized eyes: a cross sectional study Retina. 2015;35:19-20.

11. Budenz DL. Symmetry between the right and left eyes of the normal retinal nerve fibre layer measured with optical coherence tomography (an AOS thesis). Trans Am Ophthalmol coherence tomography
Soc. 2008:106:252-75.

12. Lalezary M, Shah RJ, Reddy RK, et al. Prospective Retinal and Optic Nerve Vitrectomy Evaluation (PROVE) study: twelve month findings. Ophthalmology. 2014;121:1983-9.

13. Reddy RK, Lalezary M, Kim SJ, et al. Prospective Retinal and Optic Nerve Vitrectomy Evaluation (PROVE) study: findings at 3 months. Clin Ophthalmol. 2013;7:1761-9.

14. Quigley HA, Sommer A. How to use nerve fibre layer examination in the management of glaucoma. Trans Am Ophthalmol SOc. 1987;85:254-72.

15. Mwanza JC, Durbin MK, Budenz DL, Cirrus OCT Normative Database Study Group. Interocular symmetry in peripapillary retinal nerve fibre layer thickness measured with the Cirrus HD-OCT in healthy eyes. Am J Ophthalmol. 2011:151:514-21.
16. Lentschener C, Benhamou D, Niessen F, et al. Intra-ocular pressure changes during gynaecological laparoscopy. Anaesthesia. 1996;12:1106-8.

17. Hwang JW, Oh AY, Hwang DW, et al. Does intraocular pressure increase during laparoscopic sugeries? It depends on anesthetic drugs and the surgical position. Surg Laparosc Endosc Percutan Tech. 2013;23:229-32.

18. Agrawal M, Dureja V, Verma AP, Kang LS. A comparative study of four combinations of anesthetic drugs for assessing the intraocular pressure changes during gynaecological laparoscopic procedures. Anesth Essays Res. 2013;7:319-25.

19. Mondzelewski TJ, Schmitz IW, Christman MS, et al. Intraocular pressure during robotic-assisted laparoscopic procedures utilizing steep Trendelenburg positioning. J Glaucoma. 2015;24:399-404.

20. Sit AJ. Continuous monitoring of intraocular pressure: rationale and progress toward a clinical device. J Glaucoma. 2009;18:272-9.

21. Shen Y, Drum M, Roth $S$. The prevalence of perioperative visual Ioss in the United States: a 10-year study from 1996 to 2005 of spinal, orthopedic, cardiac, and general surgery. Anesth Analg. 2009;109:1534-45.

22. Ozcaliskan S, Ozturk F, Yilmazbas P, Beyazyldiz O. Effect of dorzolamide-timolol fixed combination prophylaxis on intraocular pressure spikes after intravitreal bevacizumab injection. Int J Ophthalmol. 2015;8:496-500. 